\title{
Thermodynamic Consideration of Electromagnetic Cavity Resonators
}

Charles H. Papas

Citation: Journal of Applied Physics 25, 1552 (1954); doi: 10.1063/1.1702386

View online: http://dx.doi.org/10.1063/1.1702386

View Table of Contents: http://aip.scitation.org/toc/jap/25/12

Published by the American Institute of Physics

A|P| | $\begin{aligned} & \text { Journal of } \\ & \text { Applied Physics }\end{aligned}$

Save your money for your research.

It's now FREE to publish with us -

no page, color or publication charges apply.
Publish your research in the Journal of Applied Physics

to claim your place in applied physics history. 
technique of double doping while the crystal is being grown is not easily applicable since exceedingly slow, uninterrupted growth rates are required to form the single crystal alloy. The process of alloying impurity metals on the surface of the semiconductor is limited in the same manner since slow cooling rates must be employed to produce a coherent single crystal regrowth layer. However, the formation of $p-n$ junctions by solid phase diffusion ${ }^{3}$ of impurities into the crystal is a process which is well suited to this new material.

Vapor phase diffusion of arsenic into p-type germanium-silicon alloys was performed in a closed quartz system. Heating for one hour at $750^{\circ} \mathrm{C}$ produced an $n$-type layer $0.18 \mathrm{mil}$ in depth in a single crystal alloy of 94 percent $\mathrm{Ge}+6$ percent $\mathrm{Si}$, whereas in

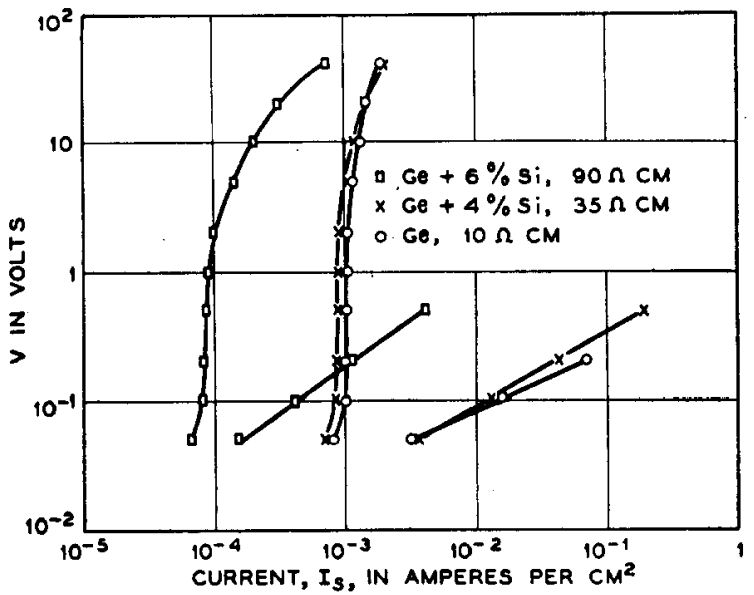

F1G, 1. Direct current voltage-current relations for junctions of area $\sim 0.03$ $\mathrm{cm}^{2}$ made by diffusion of arsenic into $\mathrm{Ge}$ and $\mathrm{Ge}-\mathrm{Si}$ alloys.

pure germanium, a $0.36-\mathrm{mil}$ layer is obtained. Since thermal conversion is as serious a problem in these alloys as it is in germanium, exacting standards of cleanliness were maintained, ${ }^{4}$ and the alloy retained its original resistivity and a high lifetime of minority carriers. For example, near a diffused junction in 90 -ohm$\mathrm{cm}$ alloy of 94 percent $\mathrm{Ge}+6$ percent $\mathrm{Si}$, the lifetime, as determined by carrier injection techniques, was in excess of $15 \mu$, the limit of the measuring apparatus.

The electrical characteristics of these diodes are shown in Fig. 1. If a comparison is made for material of equal resistivity and lifetime, there is a marked decrease in the saturation current

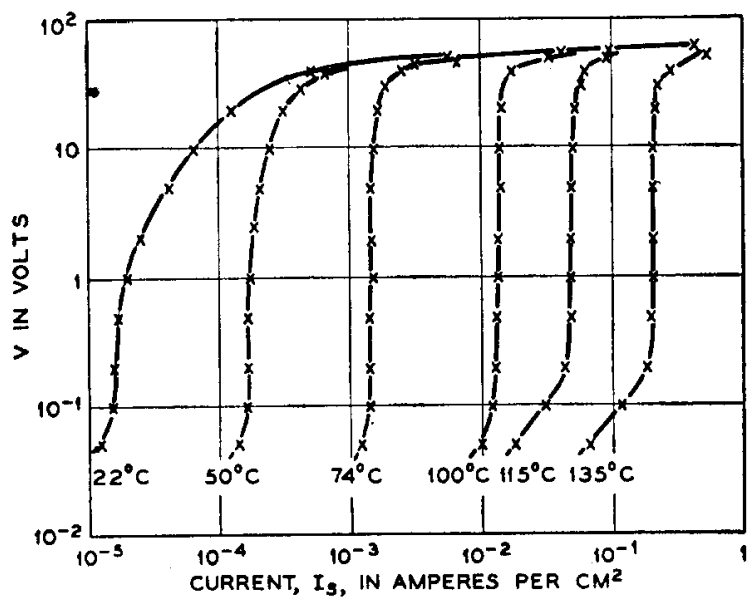

FIG. 2. Temperature dependence of dc characteristics of diode of area $0.015 \mathrm{~cm}^{2}$ made by arsenic diffusion into 95 percent $\mathrm{Ge}+5$ percent $\mathrm{Si}$ alloy of $2 \mathrm{ohm}-\mathrm{cm}$ resistivity.
$I_{\text {. }}$ as the silicon content of the alloy is increased. For example, in Fig. $2, I_{e}$ in an alloy of 95 percent $\mathrm{Ge}+5$ percent $\mathrm{Si}$ is about 02 times smaller than in pure germanium diodes, and this factor is maintained as the ambient temperature is raised to $135^{\circ} \mathrm{C}$. Infrared absorption measurements indicate an energy gap of $0.8 \mathrm{ev}$ for this alloy which is in good agreement with the observed reduction in $I_{s}$.

Experimental junction transistors have been made with the $\mathrm{Ge}-\mathrm{Si}$ alloys using diffused junctions for the emitter and collector. The base contacts were made by electroplating with rhodium and overplating with gold or soldering directly to the Ge-Si alloy with a $\mathrm{Pb}$-In solder. For ease of fabrication, the base layers were about 5 mils wide, and the observed $\alpha$ 's (which were in excess of 0.9 ) were probably limited by recombination in the base layer.

1 Levitas, Wang, and Alexander, Phys. Rev. 95, 846 (1954), also presented at American Institute of Mining Engineers Conference in New York, N. Y. February, 1954; Davis, Demars, Rubin, and Straub, Phys. Rev. 95, 597 (1954) ; E. R. Johnson and S. M. Christian, Phys. Rev. 95, 597 (1954).

In this paper, all percentages are expressed as atomic percentages.
G. L. Pearson and C. S. Fuller, Proc. Inst. Radio Engrs, 42, 760 (1954).

- R. A. Logan, Phys. Rev. 91, 757 (1953).

\section{Thermodynamic Consideration of Electromagnetic Cavity Resonators*}

\section{Charles H. Papas}

California Institute of Technology, Pasadena 4, Californio

(Received June 3, 1954)

TF a cavity resonator has a "simple shape" and is filled with a 1 homogeneous, isotropic medium, to calculate its resonant frequencies and mode functions is a straightforward task in principle. However, it is sometimes of interest to determine the oscillatory properties of a cavity that differs by a small amount in one or more of its physical characteristics from a cavity the oscillatory properties of which are known. For example, the calculation of resonant frequency shift accompanying the introduction of a small foreign body into the cavity's volume is necessary in certain measurement techniques.

A most general formula which allows the calculation of the shift in resonant frequency produced by a foreign body of given size, shape, dielectric constant, and permeability was first deduced by Müller ${ }^{1}$ and several years later by Slater $^{2}$ who used a different derivation.

It is the purpose of this letter to invite attention to a most simple derivation of the Müller-Slater formula based on thermodynamic considerations. The derivation consists of first recognizing that the change of the resonant field configuration produced by a small foreign body is adiabatic, and then applying the Boltzmann-Ehrenfest adiabatic theorem. The change produced by a foreign body is said to be adiabatic when it leaves the number of nodal surfaces unchanged. If the region occupied by a foreign body is small compared to spatial variations of the field, the undisturbed field is homogeneous throughout the volume and the disturbing influence of the foreign body is not sufficiently strong to force a jump from the original mode to an adjacent one. Hence, the restriction that the foreign body be small in the above sense is tantamount to limiting the allowable changes to adiabatic ones.

According to the Boltzmann-Ehrenfest ${ }^{3}$ adiabatic theorem, if the state of any oscillating system is changed adiabatically, the product of the period and the time average energy remains invariant. That is,

$$
W \tau=\text { constant, }
$$

where $W=$ time average energy, $\tau=2 \pi / \omega=$ period, $\omega=$ angular frequency. It follows from Eq. (1) that

$$
\frac{\delta W}{W}=\frac{\delta \omega}{\omega} \text {. }
$$


The change in time average energy produced by the foreign body is $\delta W$, and $\delta \omega$ is the corresponding change of resonant frequency. If the foreign body is placed at a position within the cavity where the electric field is $E$ and the magnetic field is $H$, and thereby becomes endowed with a magnetic moment $m$ and an electric moment $p$, the change in time average energy can be written as

$$
\delta W=-\frac{1}{4} m^{*} \cdot H-\frac{1}{2} p^{*} \cdot E,
$$

where the asterisk indicates the conjugate complex value of the quantity to which it is attached. And it follows from Eqs. (2) and (3) that

$$
-\frac{\delta \omega}{\omega}=\frac{m^{*} \cdot H+p^{*} \cdot E}{4 W} .
$$

This is the Müller-Slater formula. It applies to anisotropic foreign bodies as well as to isotropic ones. ${ }^{4}$

1 J. Müller, Hochfrequenztechnik und Elektroakustik 54, 157-161 (1939)

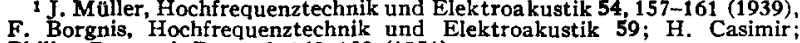
Fhilips Research Repts 6, 162-182 (1951).

Philips Research Repts 6, 162-182 (1951). Vlater, Microwave Electronics (D. Van Nostrand Company, Inc. New York, 1950), Chapter 4.

\& Y Yrillouin, Les Tenseurs (Dover Publications, New York, 1946) pp. 183-185.

* This research was supported by the Office of Naval Research

1C. H. Papas, Technical Reports No. 3 and No. 5, Department of Electrical Engineering, California Institute of Technology.

\section{Errata: Modified Luneberg Lens \\ [J. Appl. Phys. 25, 855 (1954)]}

A. S. GuTMaN
Air Force Cambridge Research Center, Cambridge, Massachusetts

THE equation in the third paragraph, first column, page 85.5 should be corrected to read

Change Eq. (3) to read

$$
n=\left[2-(r / a)^{2}\right]
$$

$$
n=(1 / f)\left(a^{2}+f^{2}-r^{2}\right)^{4} .
$$

The second group of equations in the first column on page 857 should read as follows:

$$
\begin{aligned}
& K_{1}=-f, \\
& K_{2}=a \cos \alpha, \\
& K_{3}=0, \\
& K_{4}=a \sin \alpha .
\end{aligned}
$$

Change the equation following the above group to read

$$
x=-f \cos (c t / f)+a \cos \alpha \sin (c t / f) .
$$

In the last equation of the first column on page 857 , the \pm sign should be changed to a minus sign.

Acknowledgment is made to Mr. George D. M. Peeler of the Naval Research Laboratory, Washington, D. C., for directing the attention of the author to these errata.

\section{Comments on Gumowski's Letters on Summation of Slowly Converging Series}

$$
\text { E. L. Chu }
$$

W. W. Hansen Laboratories of Physics,

Stanford University, Stanford, California

(Received September 3, 1954)

$I^{1}$ $\mathbf{N}$ his first letter ${ }^{1}$ Gumowski derives a summation formula for slowly converging series, and in a second letter, ${ }^{2}$ replying to a criticism by Braun, ${ }^{3}$ he discusses his result further. He illustrates his points by applying his result to the summation of the series, $f_{n}=1 / n^{k}, k>0$.

Regarding this topic, I would like to point out that Euler's formula is applicable to a series of the above type, provided $k>1$, as exemplified in Knopp's book, ${ }^{4}$ p. 561, Ex. 226(d), (e), and (f). In applying Euler's formula to such series, the lower limit of the summation integral is 1 , not 0 . If $k \leqq 1$, the series diverge and so cannot be summed by any formula.

For series whose general term $f(x)$ has poles or other singularities at positive real nonintegral values of $x$, the summation integral will be divergent if the lower limit of integration is not properly chosen. In such cases one can first split the series and then sum it.

The essential difference between Gumowski's result and Euler's formula is that the latter formula has a remainder term, while the former has none. With no remainder term and without its asymptotic behavior having been ascertained, a summation formula may not always be meaningful, because the series in the formula is usually divergent.

It may also be mentioned that in Chapter XIII, Section 13.12 of Hardy's book, ${ }^{5}$ one operational and several other different methods of deriving Euler's formula are described, and the use of a proper lower limit for the integral in the formula, to avoid divergence difficulty, is discussed with particular clarity.

1 I. Gumowski, J. Appl. Phys. 24, 1068 (1953).

2 . Gumowski, J. Appl. Phys. 25, 133 (1954).

4 K. K. Braun, J. Appl. Phys, 25, 132 (1954). Limited, London, England, 1946)

Limited, London, England, 1946). (Oxford University Press, New York, 1949).

\section{Experimental Studies of Betatron Orbit Stability}

G. C. Baldwin, F. R. Elder, AND W. F. WestendorP Research Laboratory, General Electric Company, Schenectady, New York (Received August 2, 1954)

$\mathrm{N}$ the design of a betatron or synchrotron, an important parameter is the guide field exponent, defined by

$$
n=-\frac{r}{B} \frac{\partial B}{\partial r}=-\frac{\partial(\log B)}{\partial(\log r)}
$$

where $r$ is the radial position coordinate, and $B$ the flux density of the magnetic guide field. This parameter determines the relative strengths of the axial and radial restoring forces which cause focussing oscillations about the instantaneous circle, with frequencies, respectively, $n^{\frac{1}{3}}$ and $(1-n)^{\frac{1}{1}}$ times the orbital revolution frequency, $\dot{\theta}$. The guide field exponent is determined largely by the polepiece profile. Its value is chosen from considerations of relative axial and radial tube apertures or from the standpoint of facilitating gun clearance during injection. We have found no experimental data in the literature on the effect of choice of $n$ on betatron performance, since it is difficult to vary $n$ in continuously excited machines.

In order to study the behavior of electrons guided by fields of various $n$ values, experiments have been made with a small betatron of simple design. The polepiece profile generates a guide field in which $n=0.69$, independent of radius for a range of one inch on either side of the 5.25-inch equilibrium orbit radius, and the magnet is designed to accommodate a standard sealed-off doughnut employed in General Electric $15-\mathrm{Mev}$ industrial radiographic betatrons. Four concentric shading coils on each polepiece, at radii of $3.75,4.5,5.25$ and 6.00 inches, respectively, in conjunction with additional turns about the centerpieces to stabilize the orbit radius, permit linear control of $n$ through a range of $0.5<n<0.9$ when connected to adjustable taps on a current transformer excited by the main magnetizing coil current. To avoid special cooling of these coils, the machine is operated discontinuously by thyratron switching to a capacitor bank. The electron gun is pulsed by a transformer excited through a delay network by the abrupt rise of voltage across the magnetizing coils at the start of the acceleration cycle. The orbit is shifted to the target by the reduction in orbit radius caused by current passed 